\title{
Profiling the EMBRACE tile beam using GPS satellite carriers
}

\author{
A.O.H. Olofsson ${ }^{1,2}$, S.A. Torchinsky ${ }^{3}$, L. Chemin ${ }^{1}$, S. Barth ${ }^{3}$, S. Bosse ${ }^{3}$, J.-M. Martin ${ }^{1}$, W. Paule 3 , P. Picard ${ }^{3}$, \\ S. Pomarède ${ }^{3}$, P. Renaud ${ }^{3}$, C. Taffoureau ${ }^{3}$, G.W. Kant ${ }^{4}$, J.E. Noordam ${ }^{4}$, S.J. Wijnholds ${ }^{4}$, R. Keller ${ }^{5}$, and \\ S. Montebugnoli 6 \\ 1 GEPI, Observatoire de Paris, 5 place Jules Janssen, Meudon, France \\ 2 Onsala Space Observatory, Chalmers University of Technology, SE-439 92 Onsala, Sweden \\ 3 Station de radioastronomie de Nançay, 18330 Nançay, France \\ 4 ASTRON, Oude Hoogeveensedijk 4, NL-7991 PD Dwingeloo, The Netherlands \\ 5 Max-Planck-Institut fuer Radioastronomie (MPIfR), Auf dem Huegel 69, 53121 Bonn, Germany \\ ${ }^{6}$ I.N.A.F.-I.R.A., Via Fiorentina 3508/B, Medicina (BO), Italy
}

\begin{abstract}
The L2C carriers of multiple GPS satellites have been used to trace out a nearly complete beam pattern out to $45^{\circ}$ away from the main lobe centre for a horizontally mounted single EMBRACE tile. The beam was formed along its bore-sight direction, i.e., staring at the local sky zenith. The result is very close to design specifications although there is evidence for at least one side lobe rising above the achieved noise level. We have also used the older L2 carrier to estimate the system temperature, although an exact figure in addition requires knowledge of the aperture efficiency.
\end{abstract}

\section{The EMBRACE instrument}

The dense aperture plane phased array system called EMBRACE is the Electronic MultiBeam Radio Astronomy ConcEpt. Densely packed Vivaldi antenna elements are organised into tiles each consisting of 72 , single-linear polarised elements. There are two arrays being constructed. One in Westerbork consisting of 144 tiles and one in Nançay with 80 tiles. A prototype tile is undergoing characterisation testing in Nançay.

EMBRACE is a prototype being designed for developing technology needed for the mid to low frequency portion of the Square Kilometre Array (SKA) project. For more details about EMBRACE see in these Proceedings Picard et al. (2010); Wijnolds et al. (2010); Berenz et al. (2010); Monari et al. (2010); Bianchi et al. (2010)

\section{GPS - a brief introduction}

\subsection{Frequencies}

The GPS satellites transmit at a number of frequencies and while the primary carrier is at $1575 \mathrm{MHz}$, it is the L2 signal at $1227.6 \mathrm{MHz}$ which is the most useful for EMBRACE test observations. At this frequency $(\lambda=24.421 \mathrm{~cm})$, the largest dimension of the tile RF beam is about $15^{\circ}$ if the instrument is approximated with a traditional dish with a diameter equivalent to the tile side of $1068 \mathrm{~mm}$. The signal is right-hand circularly polarised which is advantageous since the projection onto a linearly polarised receiver does not change over time.

\subsection{Orbit}

The GPS satellites are in what one might call "semisynchronous" Earth orbits (SSEO), i.e., they perform two orbits in exactly one sidereal day. Thus their groundtrack will be repeated every $24 \mathrm{~h}$ and this puts constraints on which satellites will ever be available for direct overhead passes. All GPS satel lites have inclinations of about $55^{\circ}$ which is fortunate since it allows zenith passages at the Nançay latitude (about $47.4^{\circ}$ ).

The SSEO is at a large distance $(\sim 20,000 \mathrm{~km})$ which means that they are visible in a large area on the ground and that a pass takes a long time (up to $7 \mathrm{~h}$ ).

\subsection{Tracking}

The information about identity and positions of the satellites can be found from NORAD, e.g. via the webpage http:// celestrak.com/NORAD/elements/. Positions are calculated and extrapolated from so called Two Line Keplerian Elements (TLEs) which contain, among other things, orbital parameters and drag coefficients. The latter is more important for low earth orbit (LEO) satellites for which orbital decay occurs more swiftly. The TLEs are updated regularly when new telemetry is available.

At any given time, about 10 of the satellites are above the horizon in Nançay. An illustrating snapshot is shown in Fig. 1.

Furthermore, two of them pass almost directly overhead, GPS BIIR-11 and GPS BIIA-25 (maximum elevation $>89^{\circ}$ ), and another handful comes well within the HPBW. It is worth reiterating that each satellite continuously provides observation opportunities once every 24 hours.

\section{Method}

The "EMBRACE Nançay Tile" was put in a recently fabricated cradle in the fully horizontal setting, see Fig. 2. The finepointing of this setup was not known a priori. The reference ro-

a A tile retroactively modified to mimick the ultimate design chosen and shipped to Nançay in advance of standard array tiles. 


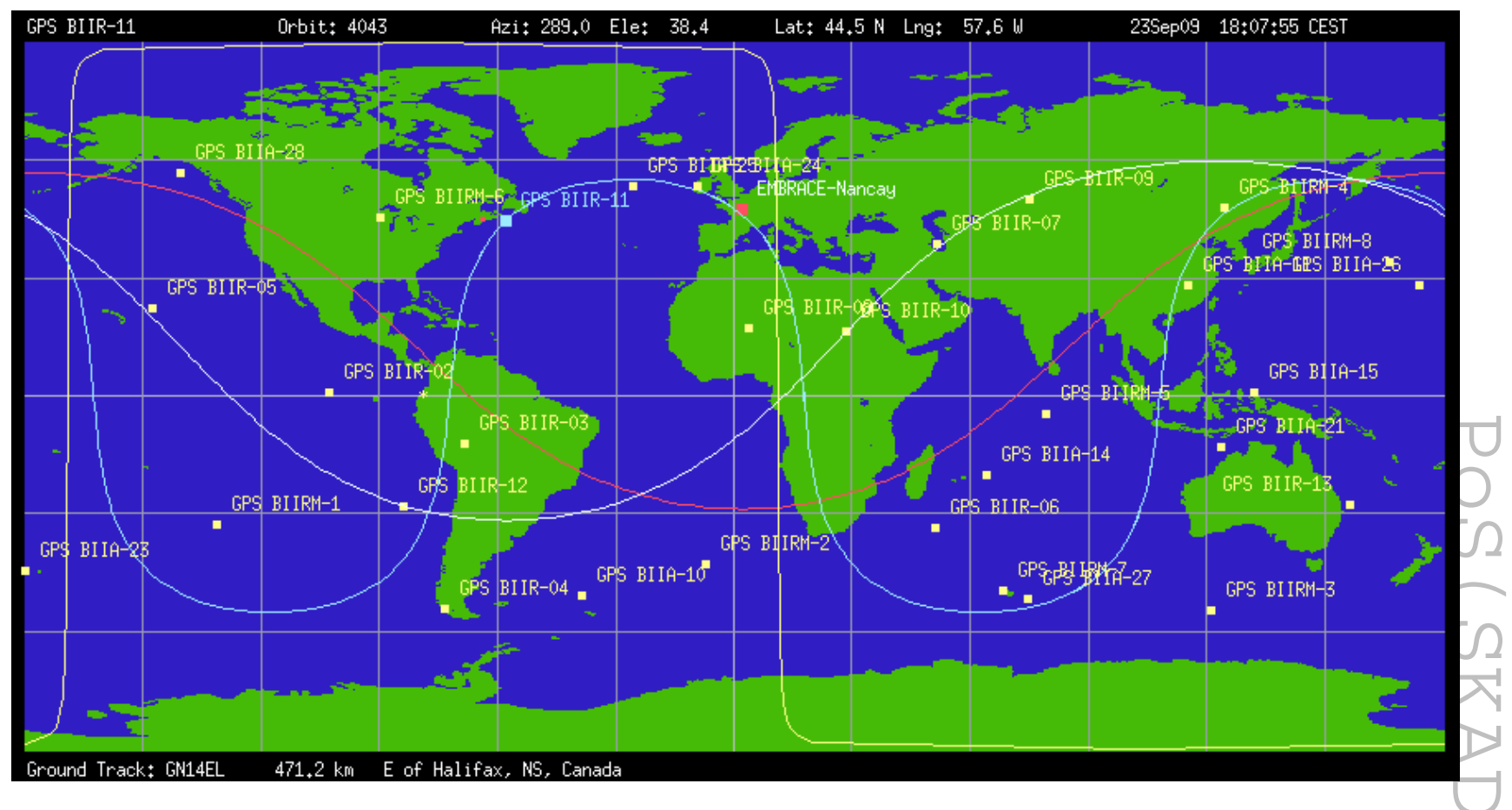

Fig. 1: Global map in Plate Carrée projection (also called Equirectangular projection) showing the ground track for two consecutive orbits of a satellite passing overhead at Nançay (light-blue line) and the instantaneous sub-satellite-position of all other operational satellites. Also of interest is the red line showing the area within which a satellite (at this orbital height) must be, such that it is above the horizon from the chosen station (EMBRACE-Nançay, coordinates lat. $47.3819^{\circ}$, long. $-2.1993^{\circ}$ )

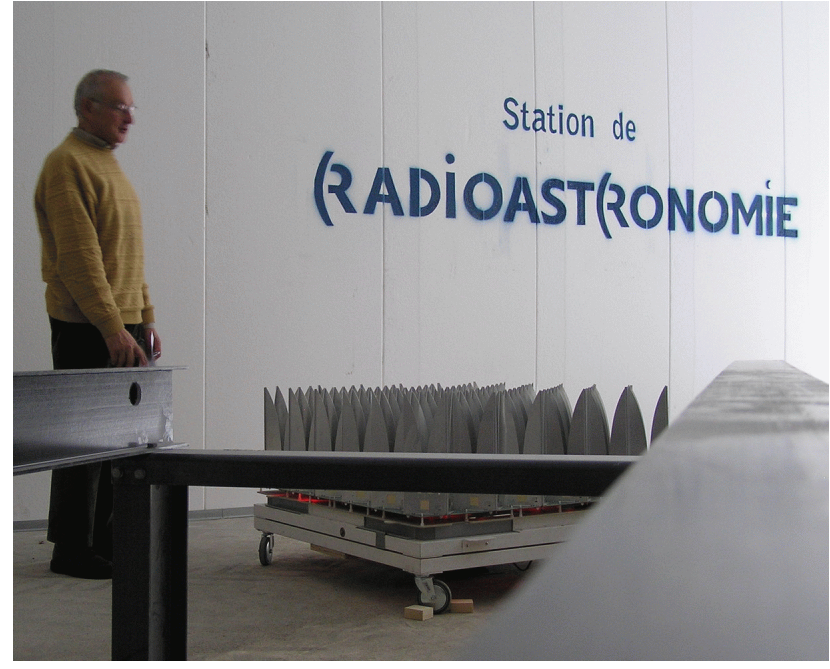

Fig. 2: The single EMBRACE tile mounted on its trolley in the horizontal position.

tation of the tile was such that the sides were along the Cardinal Directions. Due to the diagonal placement of the Vivaldi elements, that corresponds to a linear polarisation of $45^{\circ}$ with respect to an east-west line. A $30 \mathrm{MHz}$ bandpass was sampled every minute with 500 channels using a spectrum analyser that included the GPS L2 frequency at $1227.8 \mathrm{MHz}$. At the time of writing, 335 hours of observation had been performed using two different spectrum analysers as backends. To ensure that the pattern was sufficiently sampled in all directions, the tile was rotated in steps of $15^{\circ}$ between zero and $60^{\circ}$ inbetween some of the measurements.

The intensity was integrated over the central $2 \mathrm{MHz}$ as the L2C (not the weaker L2) carrier was strongly concentrated here and we attempted to avoid a pair of nearby parasitic signals.

In order to assign satellites to observed signals and to provide sky trajectories as a function of time (which was later converted to tile-centric coordinates), we employed the older, free version of "SatTrack" which is simple yet flexible since it may be customised by the user (as long as it is not spread under the copyright agreement). The positions were calculated every 30 seconds and later resampled to the points in time of the signal samples.

MatLab was later used for number crunching and visualisations once the identification phase was over.

\section{Results}

\subsection{Detections}

We first list the satellites that were unequivocally detected (but with some caveats, see below) in order of succession. The observed time signal from one measurent is shown in Fig. 3. together with satellite markers at their transit times.

Comments to table:

- The satellites with an M (for "Modernized") in their designation was found to have much stronger signals offering a very large SNR in each spectrum (and hence in each pass). 


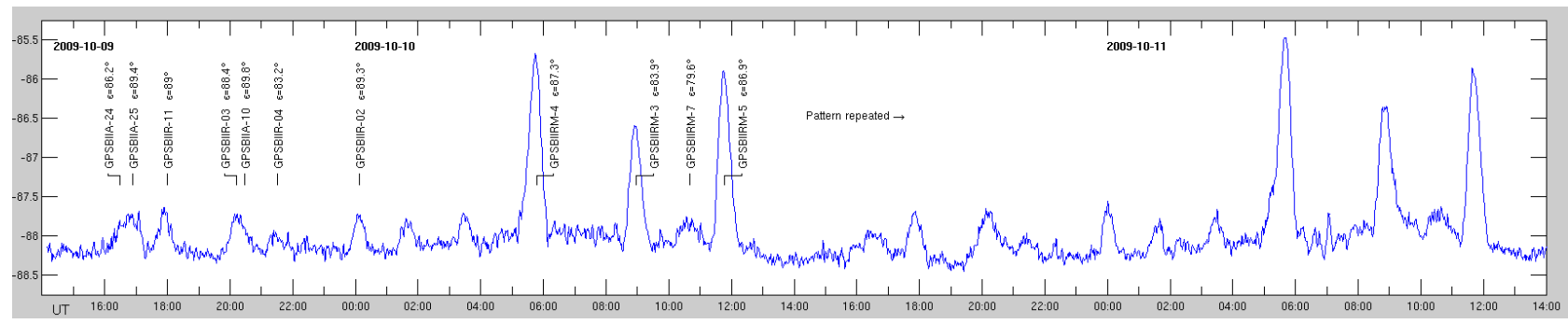

Fig. 3: A time sequence of the integrated intensity from the first multi-day measurement performed. The signal has been marginally smoothed.

\begin{tabular}{lccl}
\hline Designation & $\begin{array}{c}\text { Peak } \\
\text { elevation }\end{array}$ & $\begin{array}{c}\text { Carrier } \\
\text { type }\end{array}$ & $\begin{array}{l}\text { Signal overlap } \\
\text { (or comment) }\end{array}$ \\
\hline GPS BIIA-24 & 86.2 & L2 & w. A-25 \\
GPS BIIA-25 & 89.4 & L2 & $\begin{array}{l}\text { w. A-24, } \\
\text { dominant }\end{array}$ \\
GPS BIIR-11 & 89.0 & L2 & \\
GPS BIIR-03 & 88.4 & L2 & w. A-10, \\
& & & dominant \\
GPS BIIA-10 & 89.8 & L2 & w. R-03 \\
GPS BIIR-04 & 83.2 & L2 & marginally \\
& & & w. R-03/A-10 \\
GPS BIIR-02 & 89.3 & L2 & \\
GPS BIIRM-4 & 87.3 & L2C (strong) & \\
GPS BIIRM-3 & 83.9 & L2C (strong) & \\
GPS BIIRM-7 & 79.6 & L2C (strong) & far from \\
& & b2C (strong) & \\
GPS BIIRM-5 & 86.9 & beam centre \\
\hline
\end{tabular}

This is consistent with the stated addition of the $\mathrm{C}$ (Civil) signal also at the L2 frequency in this generation of GPS satellites (previously only used at L1 at $1575 \mathrm{MHz}$ ).

- Overlap means that one satellite had not yet completed its pass before another approached transit and the corresponding signal is a superposition of the two carriers.

- Two additional satellites, GPS BIIRM-6 and GPS BIIRM-8, were also likely detected but only through their passage through suspected side lobes, see further below.

\section{2. $2 D$ beam pattern}

In order to produce a two-dimensional beam pattern, we combined the signals from the satellites with the L2C carrier (which has a much higher signal-to-noise ratio than the older L2) taken with five different tile rotations. Figure 4 shows the trajectories for the portions of the signal sequences we used. We could make sure that these satellites had comparable intrinsic line strengths by looking at points in which their trajectories overlapped. The variations at the intersections we checked were within $10 \%$ of the beam-centre strength. Blending - i.e., when the next satellite approaches the beam pattern before the previous one has left it - was sometimes a problem and we trun-

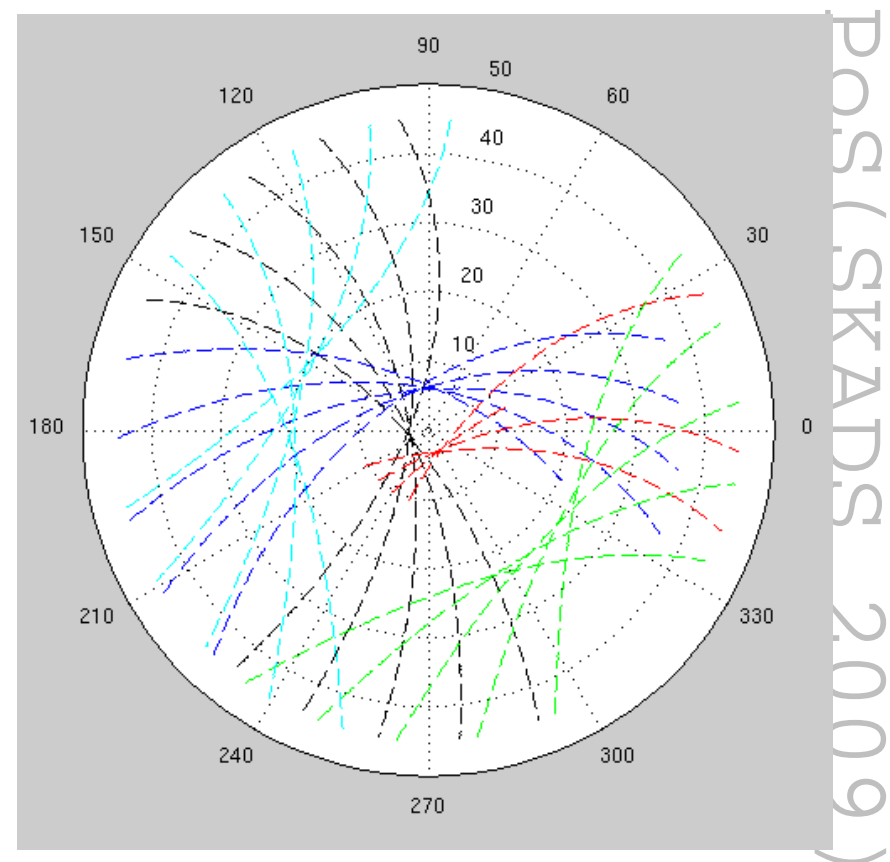

Fig. 4: Trajectories of 5 different satellites observed using 5 different tile rotations. The coordinates are fixed with respect to the tile and shown as looking onto the tile where $\phi=0$ is along the local $x$-direction (along a tile side incresing to the right). With the tile in the reference postion, the $x$-axis is parallel to an W-E line, increasing to the east.

cated the signal in these cases. It is important to note that while blending effects can produce artifacts, it cannot falsely produce a flatter - or better - pattern. Thus our results are to be considered a worst-case scenario.

We have neglected to take into account any variation of transmitter antenna gain when the satellite are farther from zenith. Even if we assume a "worst-case" scenario, in which the transmitter has Gaussian beam which is pointing to the nadir (directly down-looking) as seen from the satellite, we estimate that the HPBW would be $\gtrsim 50^{\circ \mathrm{b}}$ while the ground-station would only be $10^{\circ}$ away from nadir when the satellite is at $45^{\circ}$ elevation in the local sky. Nor would the increased path length away from zenith impose a relevant change; the received power has a square dependence on the distance but the high orbit ensures that the difference is only $1 \%$ one full beam width $\left(13^{\circ}\right)$ away from zenith and about $15 \%$ at $45^{\circ}$ elevation.

\footnotetext{
b Based on an unconfirmed bore-sight GPS antenna gain of $11.5 \mathrm{~dB}$.
} 
However, the GPS transmitting antennae actually have beam patterns that try to minimise both these effects. As is visualised in Hurtado et al. (2001) and references therein, the centre of the main lobe - the part that subtends across the surface of the Earth - is "concave" in shape, i.e. significantly weaker in the middle compared to the directions towards the limb of the Earth.

The result can be seen in Fig. 5. A 2D circular Gaussian fit gives an HPBW of $12.7^{\circ}$ and a pointing offset of only about half a degree in the negative $x$-direction. We cannot however disentangle the contributions from the instrument trolley and the tile hardware itself due to the rotations performed between measurements. Using both $\mathrm{L} 2$ and $\mathrm{L} 2 \mathrm{C}$ satellites in one single rotation indicated an offset closer to $1^{\circ}$. A slight mis-alignment in the mount structure would also serve to widen the beam somewhat and the HPBW estimate above should be considered an upper limit.

There are two areas of interest away from the main lobe, slightly below $y=0$ and roughly symmetrical around the $y$-axis, seen as elevated regions in the Figure. It seems rather likely that at least the one at positive $x$ is a real side lobe since it is seen in several satellies and disappears when the tile is rotated so that a given trajectory no longer passes over that region.

\subsection{System temperature and aperture efficiency}

$T_{\text {sys }}$ and $\eta_{\mathrm{A}}$ can be related to the received power through

$$
\frac{T_{\text {sys }}}{\eta_{\mathrm{A}}}=\frac{P_{\mathrm{t}} G_{\mathrm{t}} S_{\text {noise }} L_{\text {pol }} A_{\text {tile }}}{4 \pi d^{2} P_{r} k B}
$$

where $S_{\text {noise }}$ is the output noise power measured over bandwidth $B, L_{\mathrm{pol}}=3 \mathrm{~dB}$ is the penalty loss for using a linearly polarised antenna to receive a circularly polarised signal, and $A_{\text {tile }}$ is the physical tile area. $P, G, t, r$, and $d$, represent Power, Gain, transmitted, received, and distance, respectively. Atmospheric loss and sky noise have been neglected.

Accurate figures for $P_{t}$ are not available in official documents as it is classified information, only minimum power flux numbers seem to be stated (to allow GPS receiver manufacturers to design in adequate SNR budgets). In a few non-official sources, a figure of $4 \mathrm{~W}$ was however mentioned for the L2 carrier in seemingly fairly approximate contexts. To obtain an independent estimate, we have used some archived high-SNR GPS observations from the Onsala $25 \mathrm{~m}$ telescope. This had the additional benefit of delivering a template for the full L2 carrier spectral shape (weaker but broader than the L2C) which was used to correct for a baseline slope in the EMBRACE data which did not encompass the signal fully.

Our current best estimate lands on a carrier strength of $P_{\mathrm{t}} \approx 2.5 \mathrm{~W}$ but caution that it could be somewhat higher. While it is certain that it was tracking a GPS satellite, the information of the exact telescope pointing at the time of aquisition was not available and we assume that it was relatively close to local zenith (GPS satellites may come within a few degrees from zenith at the Onsala latitude).

As a gauge of the L2 carrier in the EMBRACE data set, we used GPS BIIR-02 which passes very close to zenith and is

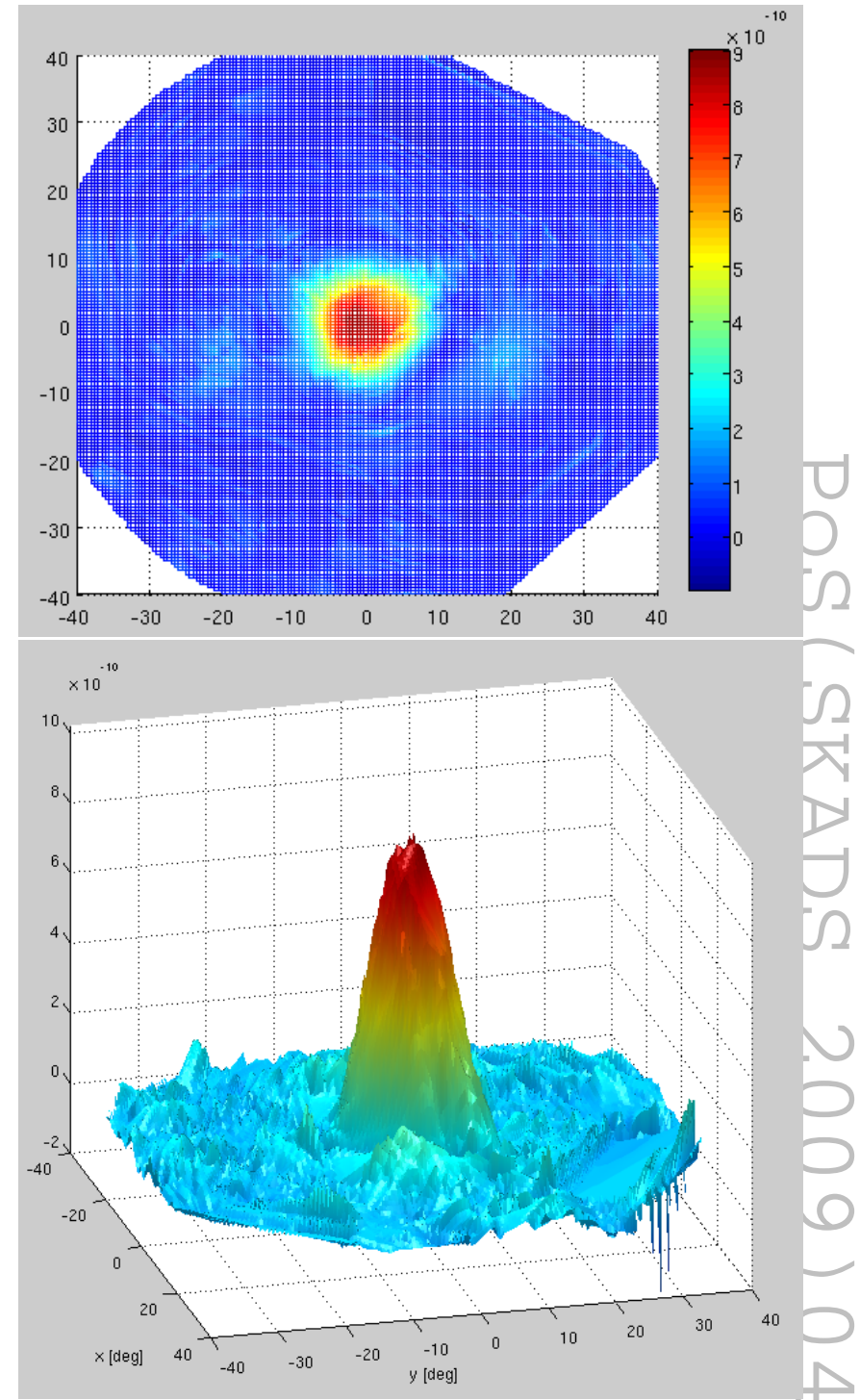

Fig. 5: a) Interpolated colour map of signal trails tracing out the beam pattern. b) $3 \mathrm{D}$ view.

entirely unaffected by blending with other signals. Its strength was in extremely good agreement between the two spectrum analysers used (the output noise baseline level was however inexplicably about a factor of 10 too high in one of them and was not trusted).

Plugging in appropriate values in the expression above, we find a $T_{\text {sys }} / \eta_{\mathrm{A}}$ of about 190 , and, should the aperture efficiency for instance turn out to be 0.5 at this frequency, we have a system temperature of slightly below $100 \mathrm{~K}$ which is consistent with expections given the various uncertainties - which may easily amount to $50 \%$ - in this simple estimate.

\subsection{Summary}

Detailed beam characterisation using GPS satellites is feasible but crowding and signal blending would be a serious problem 
for beams larger than the EMBRACE beam, or for complex beam patterns with strong side lobes. The high orbits result in slow progression on the sky yielding higher instantaneous SNR but also rather curved trajectories. The stability and repeatability of both the tile and the GPS satellite signals appear excellent.

Preliminary results indicate that the tile performance is close to expectations.

Acknowledgements. Apart from the involved parties within the SKADS community, support was also provided by the Onsala Space Observatory and Chalmers University of Technology in the form of dedicated work time and technical consultation as well as some GPS measurements taken with the $25 \mathrm{~m}$ dish.

\section{References}

Berenz, T., et al., "Mixed signal transportation for the EMBRACE antenna tiles," 2010, in Proc. Wide Field Science and Technology for the SKA, Limelette, Belgium, S.A. Torchinsky et al. (eds), ASTRON, ISBN 978-90805434-5-4

Bianchi, G., et al., "EMBRACE Local Oscillator distributor," 2010, in Proc. Wide Field Science and Technology for the SKA, Limelette, Belgium, S.A. Torchinsky et al. (eds), ASTRON, ISBN 978-90-805434-5-4

Hurtado, M., Lorente, H., \& Muravchik, C. 2001, IEEE Trans. Instrum. Meas., 50, 846

Monari, J. et al., "EMBRACE receiver design" 2010, in Proc. Wide Field Science and Technology for the SKA, Limelette, Belgium, S.A. Torchinsky et al. (eds), ASTRON, ISBN 97890-805434-5-4

Picard, P. "An overview of EMBRACE station processing," 2010, in Proc. Wide Field Science and Technology for the SKA, Limelette, Belgium, S.A. Torchinsky et al. (eds), ASTRON, ISBN 978-90-805434-5-4

Wijnholds, S.J., et al., "EMBRACE: First Experimental Results with the Initial $10 \%$ of a 10,000 Element Phased Array Radio Telescope," 2010, in Proc. Wide Field Science and Technology for the SKA, Limelette, Belgium, S.A. Torchinsky et al. (eds), ASTRON, ISBN 978-90805434-5-4 